\title{
Preference Learning for Affective Modeling
}

\author{
Georgios N. Yannakakis \\ Center for Computer Games Research \\ IT University of Copenhagen \\ Rued Langgaards Vej 7, DK-2300 Copenhagen S \\ yannakakiseitu.dk
}

\begin{abstract}
There is an increasing trend towards personalization of services and interaction. The use of computational models for learning to predict user emotional preferences is of significant importance towards system personalization. Preference learning is a machine learning research area that aids in the process of exploiting a set of specific features of an individual in an attempt to predict her preferences. This paper outlines the use of preference learning for modeling emotional preferences and shows the methodology's promise for constructing accurate computational models of affect.
\end{abstract}

\section{Introduction}

An alternative experimental approach for modeling emotional responses is presented in this paper. Specifically, the aim is the construction of computational models that can accurately predict users' emotional responses expressed as preferences. Preference learning [3,5] techniques are combined with feature set selection methods permitting the construction of preference affective models given suitable individual subject characteristics and reported emotions.

To construct affective models this way, multi-modal interaction data are collected through human survey studies. The innovation of the proposed experimental setup lies in the way human expressed response is collected. Test subjects are asked to express pairwise emotional preferences of two variants of the interactive system under investigation. This approach of eliciting emotion is named comparative affect analysis. Affective models are constructed using preference learning applied to statistical features derived from user input (e.g. physiological signals) measured during interaction and user self-report preference data. The outputs of the constructed models are real numbers that match expressed preferences of users with a high accuracy. Suitable input feature sets are chosen using various feature selection schemes. The paper outlines four alternative instance preference learning algorithms (large margin classifiers, metalarge margin classifiers, gaussian processes and evolving neural networks) and three different feature selection methods (n-best, sequential forward selection, perceptron feature selection).

Several computer [14] and physical interactive [15, 18$20]$ game case studies have already showcased the effectiveness of the proposed methodology. The class of entertainment (or "fun") preferences has been accurately predicted through affective models in those studies using feature data derived from multiple modes of interaction: interaction between the player and the game measured through game parameters and biofeedback.

\section{Affective Modeling via Preference Learning}

The procedure followed for building accurate models of emotional preferences is illustrated in Figure 1. First, an experimental protocol is designed to elicit genuine emotional responses from users of the interactive system [19]. Then data deriving from various modes of interaction are collected through the experiment and statistical features are extracted. Given the expressed preferences and the statistical features, preference learning is applied for approximating the function between selected feature subsets - derived from efficient feature selection - and reported preferences. Accurate functions of that relationship define the required user models of preference.

The reader is referred to [18] for more details on the experimental protocol used. In the following the main phases of the affective modeling procedure are described in more depth.

\subsection{System Instrumentation}

The system under investigation is instrumented based on parameters of search identified by the designer. The controllable parameters (search space dimensions) that may have an impact to the affective state of the user need to be care- 


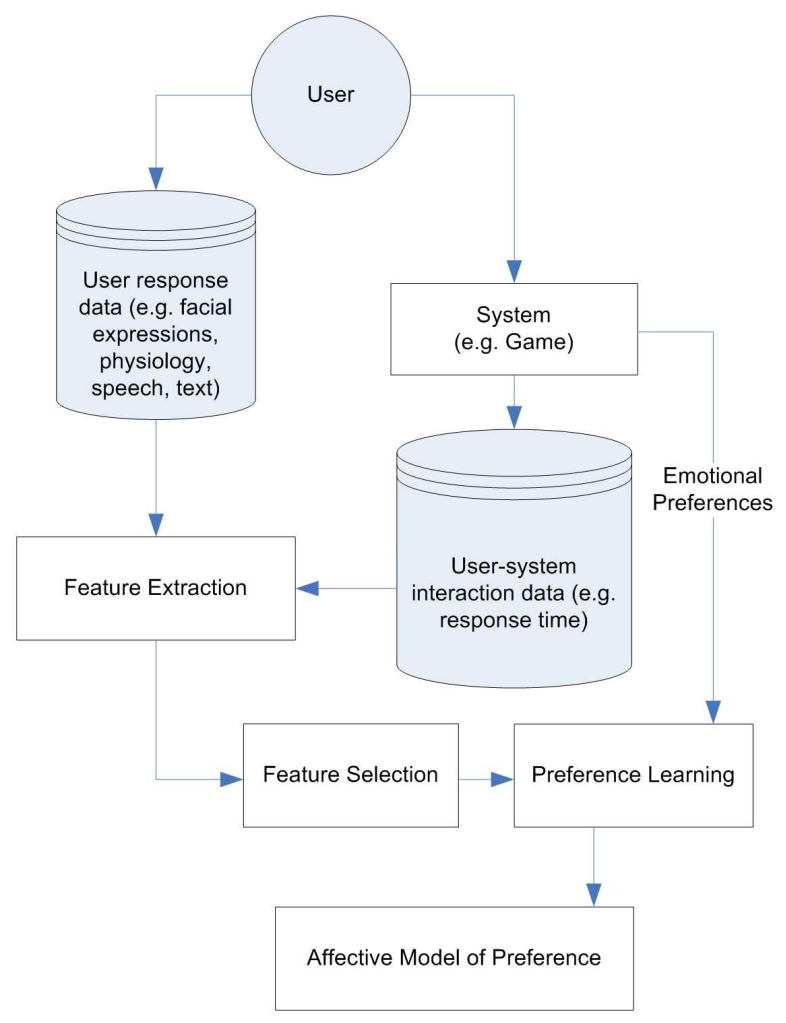

Figure 1. Methodology for constructing computational models of emotional preferences.

fully selected. The selection of the parameters is based on two main criteria: 1) potential impact of the parameter to user emotional state and 2) suitability of the parameter as a system-controller in the real-time affective loop. Pilot empirical studies may assist for automating the process of parameter selection. A controllable parameter, for instance, in a game system could be the speed of the game.

For each parameter under investigation, a number of states (e.g. 'Low', 'Average', 'High') are selected. The product of states times the parameters defines the number of points in the search space and furthermore the different system variants that will be examined. Given the proposed experimental design [18] (see Figure 2), each subject interacts with $k$ out of the $n$ variants of the selected system in all permutations of pairs. Thus, $C_{k}^{n}$ is the required number of subjects to cover all combinations of $k$ out of $n$ system variants. More specifically, each survey participant interacts with system variants in pairs (variant $A$ and variant $B$ ) differing in the levels/states of one or more of the selected controllable parameters - for a selected time window.

\subsubsection{Expressed Emotional Preferences}

Each time a pair of variants is finished, the subject is asked whether the first variant was (or felt) more $E$ than the sec-

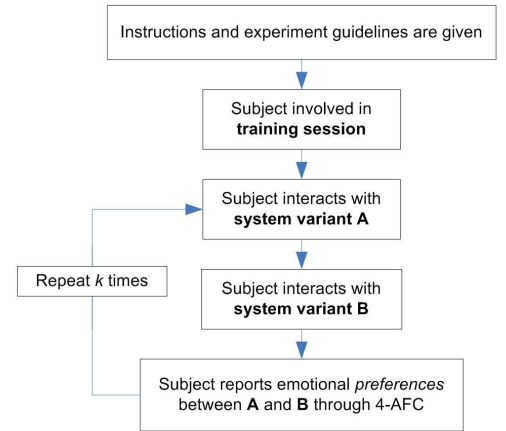

Figure 2. Experimental protocol of comparative affect analysis.

ond variant (pairwise preference). Specifically, for each completed pair of system variants $A$ and $B$, subjects report their emotional preference using a 4-alternative forced choice (4-AFC) protocol:

- $A[B]$ felt more $E$ than $B[A]$ (cf. 2-alternative forced choice);

- both felt equally $E$ or

- neither of the two felt $E$.

Where $E$ is the emotional state under investigation. For instance, $E$ could contain fun, boring, frustrating, exciting, or relaxing. The selection of the states should be based on their relevance to the test-bed interactive system and the goals of the study.

The 4-alternative forced choice (4-AFC) protocol above is used since it offers several advantages for subjective emotion capture: it minimizes the assumptions made about subjects' notions of emotions expressed (e.g. excitement, boredom, frustration) and allows a fair comparison between the answers of different subjects while also making explicit the "no preference" cases concealed by 2 -AFC. Since the focus is to construct a model relating reported emotional preferences to individual features that generalizes over the reports of different subjects 4 -AFC is preferred to e.g. a ranking - Likert scale [9] — approach. The 4-AFC and 2-AFC protocols have already been successfully utilized to provide data for building accurate computational models of reported emotional preferences [16,19].

To minimize any potential order effects each subject interacts with the aforementioned system variants in both orders. Forcing the choice of subjects generates experimental noise, in that the subject may have no significant preference for one or other of the system variants yet must nevertheless express a preference; however, insignificant order effects provide evidence that the experimental noise generated in this way is random. Statistical analysis of the effect of variant order on subjects' emotional judgement indicates 
the level of randomness in subjects' preferences. Randomness is apparent when the subject's expressed preferences are inconsistent for the pair $(A, B)$; i.e. $A \succ B$ and $B \succ A$.

\subsubsection{User Interaction Data}

Affective models can be built on several types of data collected from the users. The two main categories explored here are data obtained through the interaction between the user and the system (i.e. user-system interaction data - see Figure 1) and affective input data obtained from alternative types of user response (i.e. user response data - see Figure 1). The former includes parameters from the user's behavior derived from responses to system elements (e.g. embodied conversational agent behavior). The latter refers to a number of real-time recordings of the user's state that could be investigated and may include, among others, physiology (e.g. electrocardiogram, photoplethyspmography, galvanic skin response, respiration and jaw-electromyography), motion detection, speech and facial expression. While the amount of information about the user through multiple modalities is desired, the complication of affective modeling increases; therefore, a balance between the generated model's accuracy and computational effort has to be kept. Nevertheless the computational effort is, in part, reduced by the use of automatic feature selection.

After all data is collected, statistical feature extraction is followed (see Figure 1). The features should be carefully selected in order to reveal useful information about the user and cover the majority of the qualitative features of the signals investigated. All statistical features extracted from user interaction data together with the expressed preferences feed a preference machine learner that builds the assumed unknown function between them. Prior to preference learning, automatic feature selection is filtering the amount of information hindered in the data by selecting the minimal feature subset that yields the most accurate predictors of affect.

\subsection{Feature selection}

It is desired that the affective model constructed is dependent on a minimal number of features that yield the highest emotion prediction accuracy. The primary reasons for minimizing the feature subset are improvements of model expressiveness (interpretability) and reduction of computational effort in training and real-time performance - if the model is to be used for closing the affective loop. Therefore, feature selection is utilized to find the feature subset that yields that most accurate affective model and save computational effort of exhaustive search on all possible feature combinations. The quality of the predictive model constructed by the preference learning outlined above depends critically on the set of input data features chosen. Using the statistical features extracted from multimodal sources of subject response, a set of feature selection schemes is possible; three of them are outlined below.

\subsection{1 nBest}

nBest feature selection ranks the features used individually in order of model performance; the chosen feature set of size $\mathrm{n}$ is then the first $\mathrm{n}$ features in this ranking. The nBest method constitutes a baseline and popular technique the performance of which can be used for comparative purposes.

\subsubsection{SFS}

SFS is a bottom-up search procedure where one feature is added at a time to the current feature set. The feature to be added is selected from the subset of the remaining features such that the new feature set generates the maximum value of the performance function over all candidate features for addition. The SFS method has been successfully applied to a wide variety of feature selection problems, yielding high performance values with minimal feature subsets $[18,19$, 21].

\subsubsection{PFS}

The third method presented is Rosenblatt's perceptron as a methodology for selecting appropriate feature subsets. The algorithm of [10], which resembles artificial neural network (ANN) pruning, is adjusted to match preference learning problems here. Thus, the perceptron used employs the logistic (sigmoid) activation function in a single output neuron. The ANN's initial input vector has the size of the number of features examined. The perceptron feature selection (PFS) procedure is as follows: a) use artificial evolution to train the perceptron on the pairwise preferences - the initial input vector consists of all features extracted $\mathcal{F}$; b) eliminate all features $\mathcal{F}^{\prime}$ whose corresponding absolute connection weight values are smaller than a threshold, $T$; c) if $\mathcal{F}^{\prime}=\emptyset$ continue to $(\mathrm{d})$, otherwise use the remaining features and go to (a); d) evaluate all feature subsets obtained via preference learning.

Note that neither feature selection method is guaranteed to find the optimal feature set since neither searches all possible feature combinations. To evaluate the performance of each feature subset, the available data is randomly divided into thirds and training and validation data sets consisting of $2 / 3$ and $1 / 3$ of the data respectively are assembled. The performance of each affective model is measured through the average classification accuracy of the model in three independent runs using the 3 -fold cross-validation technique on the three possible independent training and validation data sets. 


\subsection{Preference Learning}

The problem of preference learning has recently attracted significant attention in machine learning research $[3,5]$ and has been viewed in two main ways: instance and label preference learning [5]. The focus is on the former here. Within instance preference learning (IPL) the problem consists of a set of instances $\mathcal{X}$ which are associated with a total or partial order relation and a set of pairwise preference relations $\mathcal{D}$ on the instances. In contrast to supervised learning, training instances are not specified by target values but rather by pairwise preferences between them. The instances are typically characterized by a set of attributevalue features. The goal is to learn a relation amongst the instances that satisfies those pairwise preferences. Specifically, IPL, in its general form, learns a preference relation $\mathcal{P}_{x} \subseteq \mathcal{X} \times \mathcal{X}$ for any instance $x \in \mathcal{X}$ given the pairwise preferences $\mathcal{D}=\left\{v_{k} \succ u_{k} \mid k=1, \ldots, m\right\}$. Here, $v_{k} \succ u_{k}$ means that the instance $v_{k}$ is preferred to $u_{k}$ (it is assumed in the following that $v$ 's are always preferred to $u$ 's). Previous work on this area has presented approaches that learn a utility function which assigns a score to each instance [7, 8]. Moreover, algorithms based on large margin classifiers (see $[1,4,7]$ among others) and gaussian processes [2] for preference learning have been proposed and applied to a series of dissimilar instance preference learning case studies.

The proposed approach to affective modeling is based on selecting a (constrained) minimal subset of individual features and constructing a quantitative user model that predicts the subject's reported emotional preferences. The key assumption is that a numerical value, $e$, of the reported emotion, $E$, which models the subject's affective state, is an unknown function of individual features which a machine learning mechanism can learn. The subject's expressed preferences constrain but do not specify the values for the emotional states investigated. Furthermore it is assumed that the subject's expressed preferences are consistent.

Constraint satisfaction algorithms cannot solve the problem since a variable $e$ under the constraint $e_{A}>e_{B}$ for any two given variants $A$ and $B$ has no specific domain values. Likewise, any machine learning which is based on learning a target output is inapplicable since target outputs are unknown. By the use of a ranking approach (e.g. Likert scale) numerical values for the $e$ variable could be made available; however, ranking is an undesired method for the self-report design of comparative affect analysis for the disadvantages mentioned in Section 2. Preference learning [3] is the only applicable type of machine learning for this constrained classification problem. There are several techniques that learn from a set of pairwise preferences such as algorithms based on support vector machines [4], gaussian processes [2] and evolving artificial neural networks (ANNs).
Overall, for the affective modeling problem under investigation the mechanism attempts to approximate a function that predicts whether $u_{1} \succ u_{2}$ holds, where $u_{1}, u_{2}$ represent particular instances of data features, for any $u_{1}, u_{2} \in \mathcal{X} \subset$ $\mathcal{R}^{d}$ given:

- a set of $d$ features (e.g. features extracted from usersystem interaction data or physiology) characterizing an interaction session (or interaction instance).

- a set of $n$ training instances $\mathcal{X}=\left\{x_{i} \mid i=1, \ldots, n\right\}$ comprising vectors of the measured values of those features for the (various) system variants.

- a set of $m$ pairwise preferences $\mathcal{D}=\left\{v_{k} \succ u_{k} \mid k=\right.$ $1, \ldots, m\}$ in which subjects report which of two variants they preferred $\left(u_{k}, v_{k} \in \mathcal{X}\right.$ are the feature-value vectors of the specific feature instances concerned).

Four instance preference learning mechanisms are outlined in the following: the large margin algorithm (LMA) [4], an algorithm based on LMA but using a non-linear utility function (meta-LMA), IPL with gaussian processes [2] and evolving ANNs [22].

\subsubsection{Large Margin Algorithm}

The Large Margin Algorithm (LMA) [4] is based on fundamental theory of Support Vector Machines (SVMs) and constitutes a baseline linear preference learning approach. LMA has been successfully applied in routing problems where, among many approaches, it even outperforms evolving ANNs [4]. This algorithm restricts its investigation to subjective emotional preference functions $(e)$ which are linear combinations of individual features $f$, i.e. $e(f)=$ $f \cdot w$. The vector $w=\left(w_{1}, w_{2}, \ldots, w_{d}\right)$ represents positive weight variables for the $d$ features investigated (i.e. a linear classifier) being optimized by the algorithm.

The goal is to obtain $e\left(v_{k}\right)>e\left(u_{k}\right)$ since the subject prefers a system variant with the feature vector $v_{k}$ over one with feature vector $u_{k}$ for each pairwise preference comparison $k$. Let $f_{k}=u_{k}-v_{k}, k=1, \ldots, m$; the classifier with large margin can then be obtained by solving the following linear programming problem using (for instance) the Simplex algorithm [11].

$$
\begin{array}{ll}
\text { minimize } & \sum_{j=1}^{d} w_{j} \\
\text { subject to } & w \cdot f_{k} \geq 1, k=1, \ldots, m \\
& w_{j} \geq 0, j=1, \ldots, d
\end{array}
$$




\subsubsection{Meta-LMA}

This is an algorithm inspired by the LMA algorithm sharing the same goal $\left(e\left(v_{k}\right)>e\left(u_{k}\right)\right)$ and the principal assumption that the subjective emotional preference function (e) is a linear combination of individual features. According to the meta-LMA algorithm the weight vector $w=$ $\left(w_{1}, w_{2}, \ldots, w_{d}\right)$ of $d$ selected features is adjusted to solve the following linear programming problem:

$$
\begin{array}{ll}
\text { maximize } & \frac{1}{m} \sum_{k=1}^{m} g\left(e\left(f_{k}\right), \epsilon\right) \\
\text { subject to } & e\left(f_{i}\right) \geq \delta, i=1, \ldots, m
\end{array}
$$

where $e\left(f_{k}\right)=e\left(v_{k}\right)-e\left(u_{k}\right), \delta \in[0,1]$ is the significance level of the preference ( $\delta=0.05$ in most experiments presented in the literature), $g\left(e\left(f_{k}\right), \epsilon\right)=1 /\left(1+e^{-\epsilon e\left(f_{k}\right)}\right)$ is the logistic (sigmoidal) function and $\epsilon=30$ if $e\left(f_{k}\right)>0$ and $\epsilon=5$ if $e\left(f_{k}\right)<0$. Both the sigmoidal shape of the objective function and its selected $\epsilon$ values are inspired by its successful application as a fitness function in neuroevolution preference learning problems (see section 2.3.4). Meta-LMA can be seen as a LMA variant enhanced with a non-linear, sigmoid, objective function under maximization.

\subsubsection{Bayesian Learning}

As in LMA and meta-LMA, the main idea of the Bayesian Learning (BL) approach is that there is an unobservable function $e(x)$ associated with each instance (subject) $x$ and that these function values express the preference relations observed in the data set. We utilize the approach of Williams and Rasmussen [13], who assume that the function values are random variables in a zeromean Gaussian process. The process can be estimated using the covariance matrix. The covariance between the $e$ function values that correspond to instances $x_{i}$ and $x_{j}$ can be defined by the Gaussian kernel and the elements of the $n \times n$ covariance matrix $\mathcal{G}$ are given by $\mathcal{G}=K\left(x_{i}, x_{j}\right)=\exp \left(-\kappa / 2 \sum_{l=1}^{d}\left(x_{i}^{l}-x_{j}^{l}\right)^{2}\right)$, where $\kappa$ is a positive constant and $x_{i}^{l}$ denotes the $l$-th individual feature value of subject $x_{i}$. Regarding the likelihood function, one may refer to the proposal of Chu and Ghahramani [2] in order to portray the preference relations. The function is defined as follows

$$
P(\mathcal{L} \mid \mathcal{G})=\prod_{k=1}^{m} P\left(v_{k} \succ u_{k} \mid e\left(v_{k}\right), e\left(u_{k}\right)\right)
$$

Based on Bayes' theorem, the posterior probability can be written as

$$
P(\mathcal{G} \mid \mathcal{L})=\frac{\mathcal{P}(\mathcal{G})}{\mathcal{P}(\mathcal{L})} \prod_{k=1}^{m} P\left(v_{k} \succ u_{k} \mid e\left(v_{k}\right), e\left(u_{k}\right)\right)
$$

Upon calculation of $P(\mathcal{G} \mid \mathcal{L})$ a gradient-based optimization algorithm can be implemented to shape the Bayesian model. The reader is refereed to [2] for further details of the BL algorithm.

\subsubsection{Evolving ANNs}

Given the high level of subjectivity of human preferences and the noisy nature of input data (e.g. sensor and hardware malfunctions), more complex non-linear functions such as ANNs might serve the purpose of constructing reliable affective models better. Thus, feedforward multilayered neural networks for learning the relation between the selected user features (ANN inputs) and the investigated emotional values (ANN outputs) can be utilized. Since there are no prescribed target outputs for the learning problem (i.e. no differentiable output error function), gradient-based algorithms such as back-propagation are inapplicable. Learning is achieved through artificial evolution.

A genetic algorithm (GA) can be implemented to construct a function to match the reported emotional preferences. The GA uses a fitness function that measures the difference between the subject's reported preferences and the relative magnitude of the corresponding ANN model output values $e$. While several different GA variants [6] (e.g. steady-state GA) are applicable for the problem the example algorithm outlined here is generational GA.

The ANN is itself evolved. Different evolving ANN schemes with respect to GA representation (connection weights, augmenting topologies [12]) and architecture (feedforward, recurrent) are again possible. In the algorithm presented here, the ANN topology is fixed and the GA chromosome is a vector of ANN connection weights.

A population of ANNs is initialized randomly. Then, at each generation: (a) Each member (ANN) of the population is given two $d$-tuples (where $d$ is the number of features), one for instance $A$ and one for instance $B$ for each pair $j$ of variants compared by the subject. In each case the network returns two output values, $e_{j, A}$ and $e_{j, B}$, representing the level of the corresponding emotion in each system variant. (b) Each member of the population is evaluated via a fitness function that measures the degree of match between ANN outputs $(e)$ and subject's reported answers. A high fitness results if the ranking of $e_{j, A}$ and $e_{j, B}$ matches the expressed preference of the subjects for each variant pair $j$. (c) Parents are selected and recombined to generate offspring and genetic operators are applied. The algorithm is terminated when either a good solution is found or a large number of generations has been completed. The reader is refereed to $[20,21]$ for further details on the neuro-evolutionary preference learning mechanism. 


\section{Discussion}

This paper outlines the use and showcases the promise of preference learning for building accurate models of affect in interactive systems. An experimental protocol named comparative affect analysis is proposed for efficiently capturing human response of preference. Then statistical features are extracted from data derived from various modalities of system-user interaction and automatic feature selection is applied to reduce the dimensionality of the search space. Various algorithms of preference learning can be utilized to construct the unknown function between user characteristics and emotional preferences. Four dissimilar preference learning algorithms and three different feature selection schemes are presented as examples in this paper.

Successful applications of the methodology include the construction of accurate fun predictors in simple screenbased $[14,17]$ and augmented reality game systems [15, 18-20]. Preliminary results have also shown the scalability of the method in building successful computational models of various emotional preferences (e.g. relaxation, frustration and anxiety) in commercial-standard interactive systems. These models can be used for adapting the interactive system to generate user emotional states desired by the system designer. One can then alter the interactive experience by adjusting the system control parameters investigated, thereby, efficiently closing the affective loop.

\section{References}

[1] A. Bahamonde, G. F. Bayón, J. Díez, J. R. Quevedo, O. Luaces, J. J. del Coz, J. Alonso, and F. Goyache. Feature subset selection for learning preferences: a case study. In Proceedings of the twenty-first international conference on Machine Learning, pages 49-56. ACM Press, 2004.

[2] W. Chu and Z. Ghahramani. Preference learning with gaussian processes. In Proceedings of the $22^{\text {nd }}$ International Conference on Machine Learning (ICML-2005), 2005.

[3] J. Doyle. Prospects for preferences. Computational Intelligence, 20(2):111-136, May 2004.

[4] C.-N. Fiechter and S. Rogers. Learning subjective functions with large margins. In ICML '00: Proceedings of the Seventeenth International Conference on Machine Learning, pages 287-294, San Francisco, CA, USA, 2000. Morgan Kaufmann Publishers Inc.

[5] J. Fürnkranz and E. Hüllermeier. Preference learning. Künstliche Intelligenz, 19(1):60-61, 2005.

[6] D. E. Goldberg. Genetic Algorithms in Search, Optimization and Machine Learning. Addison-Wesley, Reading, MA, 1989.

[7] R. Herbrich, T. Graepel, P. Bollmann-Sdorra, and K. Obermayer. Learning preference relations for information retrieval. In Proceedings of Text Categorization and Machine Learning Workshop, ICML, pages 80-84, 1998.

[8] T. Joachims. Optimizing search engines using clickthrough data. In Proc. KDD-02, 2002.
[9] R. Likert. A technique for the measurement of attitudes. Archives of Psychology, 140:1-55, 1932.

[10] M. Mejia-Lavalle and G. Arroyo-Figueroa. Power System Database Feature Selection Using a Relaxed Perceptron Paradigm. In Proceedings of 5th Mexican International Conference on Artificial Intelligence, LNCS, pages 522-531. Springer Berlin/Heidelberg, 2006.

[11] C. H. Papadimitriou and K. Steiglitz. Combinatorial Optimization: Algorithms and Complexity. Prentice-Hall, Englewood Cliffs, NJ, 1982.

[12] K. Stanley, B. Bryant, and R. Miikkulainen. Real-time evolution in the NERO video game. In G. Kendall and S. M. Lucas, editors, Proceedings of the IEEE Symposium on Computational Intelligence and Games, pages 182-189, Essex University, Colchester, UK, 4-6 April 2005.

[13] C. K. I. Williams and C. E. Rasmussen. Gaussian processes for regression. Advances in Neural Information Processing Systems , pages 598-604, 1996.

[14] G. N. Yannakakis and J. Hallam. Towards Capturing and Enhancing Entertainment in Computer Games. In Proceedings of the $4^{\text {th }}$ Hellenic Conference on Artificial Intelligence, Lecture Notes in Artificial Intelligence, volume 3955, pages 432-442, Heraklion, Greece, May 2006. Springer-Verlag.

[15] G. N. Yannakakis and J. Hallam. Entertainment Modeling in Physical Play through Physiology beyond Heart-Rate. In A. Paiva, R. Prada, and R. Picard, editors, Proceedings of the International Conference on Affective Computing and Intelligent Interaction, LNCS 4738, pages 256-267, Lisbon, Portugal, September 2007. Springer-Verlag.

[16] G. N. Yannakakis and J. Hallam. Game and Player Feature Selection for Entertainment Capture. In Proceedings of the IEEE Symposium on Computational Intelligence and Games, pages 244-251, Hawaii, USA, April 2007. IEEE.

[17] G. N. Yannakakis and J. Hallam. Modeling and augmenting game entertainment through challenge and curiosity. International Journal on Artificial Intelligence Tools, 16(6):981999, December 2007.

[18] G. N. Yannakakis and J. Hallam. Entertainment Modeling through Physiology in Physical Play. International Journal of Human-Computer Studies, 66:741-755, October 2008.

[19] G. N. Yannakakis, J. Hallam, and H. H. Lund. Entertainment Capture through Heart Rate Activity in Physical Interactive Playgrounds. User Modeling and User-Adapted Interaction, Special Issue: Affective Modeling and Adaptation, 18(1-2):207-243, February 2008.

[20] G. N. Yannakakis, H. H. Lund, and J. Hallam. Modeling Children's Entertainment in the Playware Playground. In Proceedings of the IEEE Symposium on Computational Intelligence and Games, pages 134-141, Reno, USA, May 2006. IEEE.

[21] G. N. Yannakakis, M. Maragoudakis, and J. Hallam. Preference Learning for Cognitive Modeling: A Case Study on Entertainment Preferences. IEEE Systems, Man and Cybernetics; Part A: Systems and Humans, 2009. (to appear).

[22] X. Yao. Evolving artificial neural networks. In Proceedings of the IEEE, volume 87, pages 1423-1447, 1999. 